\title{
Approximation of Fixed Points for Mean Nonexpansive Mappings in Banach Spaces
}

\author{
Junaid Ahmad $\mathbb{D},{ }^{1}$ Kifayat Ullah $\mathbb{D},{ }^{2}$ Muhammad Arshad, ${ }^{1}$ and Manuel de la Sen $\mathbb{D}^{3}$ \\ ${ }^{1}$ Department of Mathematics and Statistics, International Islamic University, H-10, Islamabad 44000, Pakistan \\ ${ }^{2}$ Department of Mathematics, University of Lakki Marwat, Lakki Marwat, 28420 Khyber Pakhtunkhwa, Pakistan \\ ${ }^{3}$ Institute of Research and Development of Processes, University of the Basque Country, Campus of Leioa (Bizkaia), P.O. Box 644- \\ Bilbao, Barrio Sarriena, 48940 Leioa, Spain
}

Correspondence should be addressed to Junaid Ahmad; ahmadjunaid436@gmail.com

Received 18 April 2021; Revised 21 May 2021; Accepted 28 May 2021; Published 19 June 2021

Academic Editor: Sivasubramanian Srikandan

Copyright (C) 2021 Junaid Ahmad et al. This is an open access article distributed under the Creative Commons Attribution License, which permits unrestricted use, distribution, and reproduction in any medium, provided the original work is properly cited.

In this paper, we establish weak and strong convergence theorems for mean nonexpansive maps in Banach spaces under the Picard-Mann hybrid iteration process. We also construct an example of mean nonexpansive mappings and show that it exceeds the class of nonexpansive mappings. To show the numerical accuracy of our main outcome, we show that Picard-Mann hybrid iteration process of this example is more effective than all of the Picard, Mann, and Ishikawa iterative processes.

\section{Introduction and Preliminaries}

Suppose $\mathscr{Y}$ is a Banach space and $\varnothing \neq \mathscr{W} \subseteq \mathscr{Y}$. Consider a selfmap $S: \mathscr{W} \longrightarrow \mathscr{W}$. If an element $e_{0} \in \mathscr{W}$ exists such that $e_{0}=S e_{0}$, then we say that $e_{0}$ is a fixed point for $S$. In this manuscript, we essentially represent the set $\left\{e_{0} \in \mathscr{W}: e_{0}=S e_{0}\right\}$ by $F_{S}$. The selfmap $S: \mathscr{W} \longrightarrow \mathscr{W}$ is called contraction [1] if

$$
\|S p-S q\| \leq \alpha\|p-q\| \text {, forall } p, q \in \mathscr{W} \text { and } \alpha \in[0,1) .
$$

The selfmap $S: \mathscr{W} \longrightarrow \mathscr{W}$ is called nonexpansive if (1) holds for the value $\alpha=1$. In 1965, Browder [2] and Gohde [3] proved a fixed point theorem for a nonexpansive map $S: \mathscr{W} \longrightarrow \mathscr{W}$ under the restriction that $\mathscr{Y}$ is a uniformly convex Banach space (UCBS) and $\varnothing \neq \mathscr{W} \subseteq \mathscr{Y}$ is bounded as well as closed and convex.

In [4], Zhang provided the following class of mappings.

Definition 1. Let $\mathscr{W} \neq \varnothing$ be a subset of a Banach space. A selfmap $S: \mathscr{W} \longrightarrow \mathscr{W}$ is called mean nonexpansive if for all $p$, $q \in \mathscr{W}$ there are non-negative real numbers $a, b$ such that $a$ $+b \leq 1$, we have

$$
\|S p-S q\| \leq a\|p-q\|+b\|p-S q\| .
$$

Zhang [4] provided an existence of fixed point result for mean nonexpansive mappings in Banach space setting under the normal structure assumption. After this, Wu and Zhang [5] and Zuo [6] investigated some other elementary properties and fixed point results for these mappings. In [7], Zhou and Cui used Ishikawa [8] iteration for approximating fixed points for these maps. The main aim here is to suggest some weak and strong convergence theorems for these mappings under the Picard-Mann hybrid [9] iteration and to show by a new example of mean nonexpansive maps that it converges better than the Ishikawa [8] and Mann [10] iteration processes.

Remark 2. It is easy to observe that each nonexpansive mapping is mean nonexpansive. Once again, in this research, we shall provide a new example to show that the converse is not true in general, that is, the class of mean nonexpansive maps properly includes the class of nonexpansive maps.

In the following example, $S$ is mean nonexpansive but not nonexpansive.

Example 3 (see [6]). Consider $\mathscr{W}=[0,1]$ and set $S: \mathscr{W} \longrightarrow$ $\mathscr{W}$ by 


$$
S p=\left\{\begin{array}{lll}
\frac{p}{5} & \text { if } & p<\frac{1}{2} \\
\frac{p}{6} & \text { if } & p \geq \frac{1}{2}
\end{array}\right.
$$

The Banach [1] celebrated fixed point theorem suggests the existence and uniqueness of a fixed point for a self contraction $S: \mathscr{W} \longrightarrow \mathscr{W}$ under the restriction that $\mathscr{Y}$ is complete metric space and $\varnothing \neq \mathscr{W} \subseteq \mathscr{Y}$ is closed. Also this theorem essentially uses the Picard iteration [11] for finding this unique fixed point. Nevertheless, in the case of nonexpansive maps and hence for generalized nonexpansive maps, the Picard iteration fails to converge in the associated fixed point set. For some more literature on iterative schemes, please cite the work in [12-14]. Assume that $\mathscr{W}$ is any nonempty subset of a Banach space and $S: \mathscr{W} \longrightarrow \mathscr{W}$.

The Picard [11] iterative process is stated as:

$$
\left\{\begin{array}{l}
p_{1} \in \mathscr{W}, \\
p_{m+1}=S p_{m}, m \geq 1,
\end{array}\right.
$$

Mann [10] iterative process is stated as:

$$
\left\{\begin{array}{l}
p_{1} \in \mathscr{W}, \\
p_{m+1}=\left(1-\alpha_{m}\right) p_{m}+\alpha_{m} S p_{m}, m \geq 1
\end{array}\right.
$$

where $\alpha_{m} \in(0,1)$.

Ishikawa [8] iteration process may be viewed as a twostep Mann iteration, stated as follows:

$$
\left\{\begin{array}{l}
p_{1} \in \mathscr{W}, \\
q_{m}=\left(1-\beta_{m}\right) p_{m}+\beta_{m} S p_{m}, \\
p_{m+1}=\left(1-\alpha_{m}\right) p_{m}+\alpha_{m} S q_{m}, m \geq 1,
\end{array}\right.
$$

where $\alpha_{m}, \beta_{m} \in(0,1)$.

Khan [9] introduced the Picard-Mann hybrid iteration as follows:

$$
\left\{\begin{array}{l}
p_{1} \in \mathscr{W} \\
q_{m}=\left(1-\alpha_{m}\right) p_{m}+\alpha_{m} S p_{m} \\
p_{m+1}=S q_{m}, m \geq 1
\end{array}\right.
$$

where $\alpha_{m} \in(0,1)$.

Khan [9] provided the weak and strong convergence of the scheme (7) for the class of nonexpansive operators. Furthermore, he proved that the Picard-Mann hybrid iteration process is more effective than the Picard (4), Mann (5) and Ishikawa (6) iteration processes in the setting of nonexpansive maps. In this paper, we connect this scheme with the class of mean nonexpansive mappings, and in this way, we extend his results in the more general setting of mean nonexpansive mappings.

Now we provide some elementary definitions and results, which will be used in sequel.
Definition 4 [15]. A Banach space $\mathcal{Y}$ is called UCBS if and only if for every choice of $r \in(0,2]$, one has a $s>0$ such that for every $p, q$ in $\mathcal{Y}, 1 / 2\|p+q\| \leq(1-s)$ whenever $\|p\| \leq 1$, $\|q\| \leq 1$ and $\|p-q\|>r$.

Definition 5 [16]. A Banach space $\mathcal{Y}$ is said to satisfy the Opial's property if any weakly convergent sequence $\left\{p_{m}\right\}$ in $\mathcal{Y}$ which admits a weak limit $c \in \mathcal{Y}$, one has

$$
\limsup _{m \longrightarrow \infty}\left\|p_{m}-c\right\|<\limsup _{m \rightarrow \infty}\left\|p_{m}-c^{\prime}\right\| \text {, foreach } c^{\prime} \in \mathcal{Y}-\{c\} .
$$

Definition 6 [17]. Suppose $\mathscr{W} \neq \varnothing$ is any subset of a Banach space $\mathscr{Y}$. A selfmap $S: \mathscr{W} \longrightarrow \mathscr{W}$ is said to be endowed with the condition $(I)$ if and only if a function $L:[0, \infty) \longrightarrow[0$, $\infty)$ exists sucht that $L(0)=0$ and $L(r)>0$ for all $r>0$ and $\|p-S p\| \geq L\left(d\left(p, F_{S}\right)\right)$ for each $p \in \mathscr{W}$.

The following lemma holds, which suggests many examples of mean nonexpansive mappings.

Lemma 7. If $S$ is a selfmap and nonexpansive on a subset $\mathscr{W} \neq \varnothing$ of a Banach space. Then $S$ is mean nonexpansive.

From the definition of mean nonexpansive maps, we have the following facts.

Lemma 8. If $S$ is a selfmap and mean nonexpansive on a subset $\mathscr{W} \neq \varnothing$ of a Banach space $\mathcal{Y}$. Then $F_{S}$ is closed. Moreover, if $\mathcal{Y}$ is strictly convex and $\mathscr{W}$ is convex, then $F_{S}$ is also convex.

Theorem 9 [6]. Let $\mathscr{W} \neq \varnothing$ be a subset of a reflexive Banach space (RBS) Y having Opial property. Let $S: \mathscr{W} \longrightarrow \mathscr{W}$ be a mean nonexpansive mapping. If $\left\{p_{m}\right\} \subseteq \mathscr{W}$ be such that.

$\left(a_{0}\right)\left\{p_{m}\right\}$ converges weakly to $e_{0}$,

$\left(b_{0}\right) \lim _{m \longrightarrow \infty}\left\|S p_{m}-p_{m}\right\|=0$,

then $e_{0}=S e_{0}$.

Any UCBS can be characterized by the following way.

Lemma 10 [18]. If $\mathcal{Y}$ is a UCBS and If $\left\{s_{m}\right\}$ and $\left\{w_{m}\right\}$ are two sequences in $\mathcal{Y}$ such that $\limsup _{m \rightarrow \infty}\left\|s_{m}\right\| \leq l$, $\limsup _{m \rightarrow \infty}\left\|w_{m}\right\| \leq l$ and $\lim _{m \longrightarrow \infty}\left\|\delta_{m} s_{m}+\left(1-\delta_{m}\right) w_{m}\right\|$ $=l$ for some $l \geq 0$ and $0<q \leq \delta_{m} \leq p<1$. Then, $\lim _{m \longrightarrow \infty} \| s_{m}$ $-w_{m} \|=0$.

\section{Main Results}

The following results are the main outcome of this section. Notice that all these results improve and extend some main results of Khan [9] from the case of nonexpansive maps to case of mean nonexpansive maps.

Lemma 11. Let $\mathscr{W} \neq \varnothing$ be a convex closed subset of a UCBS $\mathscr{Y}$ and $S: \mathscr{W} \longrightarrow \mathscr{W}$ be a mean nonexpansive mapping and $F_{S}$ $\neq \varnothing$. Assume that $\left\{p_{m}\right\}$ is a sequence of Picard-Mann hybrid 
iterative process (7). Consequently $\lim _{m \rightarrow \infty}\left\|p_{m}-e_{0}\right\|$ exists for each $e_{0} \in F_{S}$.

Proof. Let $e_{0} \in F_{S}$. Then using (7), we have

$$
\begin{aligned}
\left\|q_{m}-e_{0}\right\| & \leq\left(1-\alpha_{m}\right)\left\|p_{m}-e_{0}\right\|+\alpha_{m}\left\|p_{m}-e_{0}\right\| \\
& \leq\left(1-\alpha_{m}\right)\left\|p_{m}-e_{0}\right\|+\alpha_{m}\left\|S p_{m}-e_{0}\right\| \\
& =\left(1-\alpha_{m}\right)\left\|p_{m}-e_{0}\right\|+\alpha_{m}\left\|S p_{m}-S e_{0}\right\| \\
& \leq\left(1-\alpha_{m}\right)\left\|p_{m}-e_{0}\right\|+\alpha_{m}\left(a\left\|p_{m}-e_{0}\right\|+b\left\|p_{m}-S e_{0}\right\|\right) \\
& =\left(1-\alpha_{m}\right)\left\|p_{m}-e_{0}\right\|+\alpha_{m}\left(a\left\|p_{m}-e_{0}\right\|+b\left\|p_{m}-e_{0}\right\|\right) \\
& =\left(1-\alpha_{m}\right)\left\|p_{m}-e_{0}\right\|+\alpha_{m}\left((a+b)\left\|p_{m}-e_{0}\right\|\right) \\
& =\left(1-\alpha_{m}\right)\left\|p_{m}-e_{0}\right\|+\alpha_{m}\left\|p_{m}-e_{0}\right\| \\
& \leq\left\|p_{m}-e_{0}\right\| .
\end{aligned}
$$

This implies that

$$
\begin{aligned}
\left\|p_{m+1}-e_{0}\right\| & =\left\|S q_{m}-e_{0}\right\| \\
& =\left\|S q_{m}-S e_{0}\right\| \\
& \leq a\left\|q_{m}-e_{0}\right\|+b\left\|q_{m}-S e_{0}\right\| \\
& =a\left\|q_{m}-e_{0}\right\|+b\left\|q_{m}-e_{0}\right\| \\
& =(a+b)\left\|q_{m}-e_{0}\right\| \\
& \leq\left\|q_{m}-e_{0}\right\| \\
& \leq\left\|p_{m}-e_{0}\right\| .
\end{aligned}
$$

We have showed that $\left\|p_{m+1}-e_{0}\right\| \leq\left\|p_{m}-e_{0}\right\|$. It follows that $\left\{\left\|p_{m}-e_{0}\right\|\right\}$ is nonincreasing and bounded. Thus $\lim _{m \longrightarrow \infty}\left\|p_{m}-e_{0}\right\|$ exists for each $e_{0} \in F_{S}$.

Theorem 12. Let $\mathscr{W} \neq \varnothing$ be a convex closed subset of a UCBS $\mathscr{Y}$ and $S: \mathscr{W} \longrightarrow \mathscr{W}$ be a mean nonexpansive mapping and $F_{S} \neq \varnothing$. Assume that $\left\{p_{m}\right\}$ is a sequence of Picard-Mann hybrid iterative process (7). Consequently, $\left\{p_{m}\right\}$ is bounded in $Y$ with the property $\lim _{m \rightarrow \infty}\left\|S p_{m}-p_{m}\right\|=0$.

Proof. Since the set $F_{S}$ is nonempty so we may choose any $e_{0} \in F_{S}$. By Lemma $11, \lim _{m \rightarrow \infty}\left\|p_{m}-e_{0}\right\|$ exists and $\left\{p_{m}\right\}$ is bounded. Suppose that

$$
\lim _{m \longrightarrow \infty}\left\|p_{m}-e_{0}\right\|=s .
$$

By looking in the proof of Lemma 11, one see

$\left\|q_{m}-e_{0}\right\| \leq\left\|p_{m}-e_{0}\right\|, \Rightarrow \limsup _{m \longrightarrow \infty}\left\|q_{m}-e_{0}\right\| \leq \limsup _{m \longrightarrow \infty}\left\|p_{m}-e_{0}\right\|=s$.

Now

$$
\begin{aligned}
\left\|S p_{m}-e_{0}\right\| & =\left\|S p_{m}-S e_{0}\right\| \\
& \leq a\left\|p_{m}-e_{0}\right\|+b\left\|p_{m}-S e_{0}\right\| \\
& =(a+b)\left\|p_{m}-e_{0}\right\| \\
& \leq\left\|p_{m}-e_{0}\right\| .
\end{aligned}
$$

It follows that

$$
\limsup _{m \rightarrow \infty}\left\|S p_{m}-e_{0}\right\| \leq \limsup _{m \longrightarrow \infty}\left\|p_{m}-e_{0}\right\|=s
$$

Again by looking in the proof of Lemma 11, one see

$$
\left\|p_{m+1}-q\right\| \leq\left\|q_{m}-q\right\| .
$$

It follows that

$$
s \leq \liminf _{m \rightarrow \infty}\left\|q_{m}-e_{0}\right\|
$$

From (12) and (16), we obtain

$$
s=\lim _{m \longrightarrow \infty}\left\|q_{m}-e_{0}\right\| .
$$

From (17), we have

$$
\begin{aligned}
s & =\lim _{m \longrightarrow \infty}\left\|q_{m}-q\right\| \\
& =\lim _{m \longrightarrow \infty}\left\|\left(1-\alpha_{m}\right) p_{m}+\alpha_{m} S p_{m}-e_{0}\right\| \\
& =\lim _{m \longrightarrow \infty}\left\|\left(1-\alpha_{m}\right)\left(p_{m}-e_{0}\right)+\alpha_{m}\left(S p_{m}-e_{0}\right)\right\| .
\end{aligned}
$$

Hence,

$$
s=\lim _{m \longrightarrow \infty}\left\|\left(1-\alpha_{m}\right)\left(p_{m}-e_{0}\right)+\alpha_{m}\left(S p_{m}-e_{0}\right)\right\| .
$$

Now from (11), (14) and (19) together with Lemma 10, we obtain

$$
\lim _{m \longrightarrow \infty}\left\|S p_{m}-p_{m}\right\|=0
$$

We now provide a weak convergence theorem under the assumption of the Opial's condition.

Theorem 13. Let $\mathscr{W} \neq \varnothing$ be a convex closed subset of a UCBS $\mathcal{Y}$ and $S: \mathscr{W} \longrightarrow \mathscr{W}$ be a mean nonexpansive mapping and $F_{S} \neq \varnothing$. Assume that $\left\{p_{m}\right\}$ is a sequence of Picard-Mann hybrid iterative process (7). If $y$ has the Opial property, then $\left\{p_{m}\right\}$ converges weakly to a point of of $F_{S}$.

Proof. By Theorem 12, the sequence $\left\{p_{m}\right\}$ is bounded and $\lim _{n \rightarrow \infty}\left\|S p_{m}-p_{m}\right\|=0$. Since $y$ is UCBS, it follows that $\mathcal{Y}$ is RBS. Thus one has a weakly convergent subsequence $\left\{p_{m_{i}}\right\}$ of $\left\{p_{m}\right\}$ exists with some weak limit $q_{1} \in \mathscr{W}$. By Theorem $9, q_{1} \in F_{S}$. Next we show that $\left\{p_{m}\right\}$ is weakly convergent to $q_{1}$. We may suppose that $\left\{p_{m}\right\}$ is not weakly convergent to $q_{1}$, that is, one has a weakly convergent subsequence $\left\{p_{m_{j}}\right\}$ of $\left\{p_{m}\right\}$ with a weak limit $q_{2} \in \mathscr{W}$ and $q_{2} \neq q_{1}$. Again applying Theorem $9, q_{2} \in F_{S}$. By applying Opial's condition and keeping Lemma 11 in mind, it follow that 


$$
\begin{aligned}
\lim _{m \longrightarrow \infty}\left\|p_{m}-q_{1}\right\| & =\lim _{i \longrightarrow \infty}\left\|p_{m_{i}}-q_{1}\right\| \\
& <\lim _{i \longrightarrow \infty}\left\|p_{m_{i}}-q_{2}\right\| \\
& =\lim _{m \longrightarrow \infty}\left\|p_{m}-q_{2}\right\| \\
& =\lim _{j \longrightarrow \infty}\left\|p_{m_{j}}-q_{2}\right\| \\
& <\lim _{j \longrightarrow \infty}\left\|p_{m_{j}}-q_{1}\right\| \\
& =\lim _{m \longrightarrow \infty}\left\|p_{m}-q_{1}\right\| .
\end{aligned}
$$

Hence we have seen a contradiction. Accordingly, we have $q_{1}=q_{2}$. Thus, $\left\{p_{m}\right\}$ converges weakly to $q_{1} \in F_{S}$.

The strong convergence theorem under the assumption of compactness is established as follows.

Theorem 14. Let $\mathscr{W} \neq \varnothing$ be a convex compact subset of a UCBS $\mathscr{Y}$ and $S: \mathscr{W} \longrightarrow \mathscr{W}$ be a mean nonexpansive mapping and $F_{S} \neq \varnothing$. Assume that $\left\{p_{m}\right\}$ is a sequence of Picard-Mann hybrid iterative process (7). If $\mathscr{W}$ is compact, then $\left\{p_{m}\right\}$ converges strongly to an element of $F_{S}$.

Proof. Since $\mathscr{W}$ is compact, and $\left\{p_{m}\right\} \subseteq \mathscr{W}$. One can choose a strongly convergent subsequence $\left\{p_{m_{k}}\right\}$ of $\left\{p_{m}\right\}$ such that $p_{m_{k}} \longrightarrow u$. Now we show that $S u=u$. For this

$$
\begin{aligned}
\|u-S u\| \leq & \left\|u-p_{m_{k}}\right\|+\left\|p_{m_{k}}-S p_{m_{k}}\right\|+\left\|S p_{m_{k}}-S u\right\| \\
\leq & \left\|u-p_{m_{k}}\right\|+\left\|p_{m_{k}}-S p_{m_{k}}\right\| \\
& +\left(a\left\|p_{m_{k}}-u\right\|+b\left\|u-S p_{m_{k}}\right\|\right) \\
\leq & \left\|u-p_{m_{k}}\right\|+\left\|p_{m_{k}}-S p_{m_{k}}\right\| \\
& +\left(a\left\|p_{m_{k}}-u\right\|+b\left\|u-p_{m_{k}}\right\|+b\left\|p_{m_{k}}-S p_{m_{k}}\right\|\right) \\
= & (a+b+1)\left\|u-p_{m_{k}}\right\|+(b+1)\left\|p_{m_{k}}-S p_{m_{k}}\right\| .
\end{aligned}
$$

Consequently, we obtained

$$
\|u-S u\| \leq(a+b+1)\left\|u-p_{m_{k}}\right\|+(b+1)\left\|p_{m_{k}}-S p_{m_{k}}\right\| .
$$

According to Theorem 12, we have $\lim _{k \longrightarrow \infty} \| p_{m_{k}}-S p_{m_{k}}$ $\|=0$, so applying $k \longrightarrow \infty$, we obtain $S u=u$. This shows that $u \in F_{S}$. By Lemma 11, $\lim _{m \rightarrow \infty}\left\|p_{m}-u\right\|$ exists. Consequently, $u$ is the strong limit of $\left\{p_{m}\right\}$ and element of $F_{S}$.

The strong convergence theorem without the compactness assumption is established as follows.
Theorem 15. Let $\mathscr{W} \neq \varnothing$ be a convex closed subset of a UCBS $\mathscr{Y}$ and $S: \mathscr{W} \longrightarrow \mathscr{W}$ be a mean nonexpansive mapping and $F_{S} \neq \varnothing$. Assume that $\left\{p_{m}\right\}$ is a sequence of Picard-Mann hybrid iterative process (7). Then $\left\{p_{m}\right\}$ converges strongly to an element of $F_{S}$ if and only if liminf ${ }_{m \rightarrow \infty} d\left(p_{m}, F_{S}\right)=0$.

Proof. The necessity is obvious.

Conversely, suppose that $\liminf _{m \longrightarrow \infty} d\left(p_{m}, F_{S}\right)=0$ and $e_{0} \in F_{S}$. From the Lemma 11, $\lim _{m \rightarrow \infty}\left\|p_{m}-e_{0}\right\|$ exists. Therefore $\lim _{m \longrightarrow \infty} d\left(p_{m}, F_{S}\right)=0$, by assumption. We prove that $\left\{p_{m}\right\}$ is a Cauchy sequence in $\mathscr{W}$. As $\lim _{m \rightarrow \infty} d\left(p_{m}, F_{S}\right)$ $=0$, for a given $\varepsilon>0$, there exists $r_{0} \in \mathbb{N}$ such that for each $m \geq r_{0}$,

$$
d\left(p_{m}, F_{S}\right)<\frac{\varepsilon}{2} . \Rightarrow \inf \left\{\left\|p_{m}-e_{0}\right\|: e_{0} \in F_{S}\right\}<\frac{\varepsilon}{2} .
$$

In particular inf $\left\{\left\|p_{r_{0}}-e_{0}\right\|: e_{0} \in F_{S}\right\}<\varepsilon / 2$. Therefore there exists $e_{0} \in F_{S}$ such that

$$
\left\|p_{r_{0}}-e_{0}\right\|<\frac{\varepsilon}{2}
$$

Now for $r, m \geq k_{0}$,

$$
\begin{aligned}
\left\|p_{m+r}-p_{m}\right\| & \leq\left\|p_{m+r}-e_{0}\right\|+\left\|p_{m}-e_{0}\right\| \\
& \leq\left\|p_{r_{0}}-e_{0}\right\|+\left\|p_{r_{0}}-e_{0}\right\| \\
& =2\left\|p_{r_{0}}-e_{0}\right\|<\varepsilon .
\end{aligned}
$$

This shows that $\left\{p_{m}\right\}$ is a Cauchy sequence in $\mathscr{W}$. As $\mathscr{W}$ is closed subset of a Banach space $\mathscr{Y}$, so there exists a point $e_{0}$ $\in \mathscr{W}$ such that $\lim _{m \rightarrow \infty} p_{m}=e_{0}$. Now $\lim _{m \rightarrow \infty} d\left(p_{m}, F_{S}\right)=$ 0 gives that $d\left(e_{0}, F_{S}\right)=0$. Since from Lemma 8 , we have the set $F_{S}$ a closed set in $\mathscr{W}$. Hence $e_{0} \in F_{S}$.

The below facts are essentially due to Sentor and Dotson [17].

Definition 16. Let $\mathscr{W} \neq \varnothing$ be a subset of a Banach space $\mathscr{Y}$. A selfmap $S: \mathscr{W} \longrightarrow \mathscr{W}$ is said to be endowed with the condition $(I)$ if and only if a function $L:[0, \infty) \longrightarrow[0, \infty)$ exists such that $L(0)=0$ and $L(r)>0$ for all $r>0$ and $\|p-S p\| \geq$ $L\left(d\left(p, F_{S}\right)\right)$ for each $p \in \mathscr{W}$.

The strong convergence theorem under the assumption of condition $(I)$ is established as follows.

Theorem 17. Let $\mathscr{W} \neq \varnothing$ be a convex closed subset of a UCBS $\mathscr{Y}$ and $S: \mathscr{W} \longrightarrow \mathscr{W}$ be a mean nonexpansive mapping and $F_{S} \neq \varnothing$. Assume that $\left\{p_{m}\right\}$ is a sequence of Picard-Mann hybrid iterative process (7). If $S$ is endowed with condition $(I)$, then $\left\{p_{m}\right\}$ converges strongly to an element of $F_{S}$.

Proof. From Theorem 12, we have

$$
\lim _{m \longrightarrow \infty}\left\|S p_{m}-p_{m}\right\|=0
$$


TABle 1: Strong convergence of Picard-Mann hybrid, Picard, Ishikawa and Mann iterations to the fixed point $e_{0}=0$ of $S$ in Example 19.

\begin{tabular}{lcccc}
\hline $\mathrm{m}$ & Picard-Mann hybrid (7) & Picard (4) & Ishikawa (6) & Mann (5) \\
\hline 1 & 0.90000000000000 & 0.90000000000000 & 0.90000000000000 & 0.90000000000000 \\
2 & 0.07920000000000 & 0.18000000000000 & 0.33048000000000 & 0.39600000000000 \\
3 & 0.00696960000000 & 0.03600000000000 & 0.12135225000000 & 0.17424000000000 \\
4 & 0.00061332480000 & 0.00720000000000 & 0.04456054840320 & 0.07666560000000 \\
5 & 0 & 0.00144000000000 & 0.01636263337365 & 0.03373286400000 \\
6 & 0 & 0 & 0.00600835897480 & 0.01484246010000 \\
7 & 0 & 0 & 0.00220626941554 & 0.00653068247000 \\
8 & 0 & 0 & 0.00066188082466 & 0.00287350028697 \\
9 & 0 & 0 & 0.00019856424739 & 0.00126434012626 \\
10 & 0 & 0 & 0.00005956927421 & 0.00037930203788 \\
11 & 0 & 0 & 0.00001787078226 & 0.00011379061136 \\
\hline
\end{tabular}

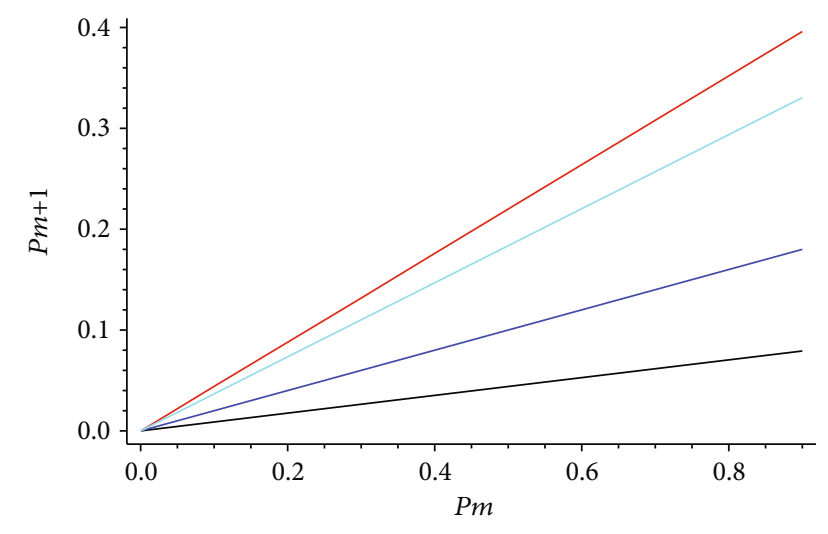

Figure 1: Behaviors of Picard-Mann hybrid (black), Picard (blue), Ishikawa (cyan) and Mann (red) iterates to the unique fixed point $e_{0}=0$ of the selfmap $S$.

Condition (I) of $S$ provides

$$
\lim _{m \longrightarrow \infty} d\left(p_{m}, F_{S}\right)=0
$$

Now all the requirements of the Theorem 15 are available, so we conclude that $\left\{p_{m}\right\}$ converges strongly to an element of $F_{S}$.

Remark 18. In the view of Lemma 7, our results contains the case of nonexpansive mappings.

\section{Example}

Now we want to provide a new example of mean nonexpansive maps.

Example 19. Let $S:[0,1] \longrightarrow[0,1]$ be defined by $S p=0$ if 0 $\leq p<1 / 515$ and $S p=p / 5$ for $1 / 515 \leq p \leq 1$. Suppose $a=1 / 4$ $=b$. Then it is easy to show $a+b \leq 1$.

Now we may consider the following cases.

(i) Suppose that $0 \leq p, q<1 / 515$. Then

$$
|S p-S q|=0 \leq a|p-q|+b|p-S q| .
$$

(ii) Suppose that $1 / 515 \leq p, q \leq 1$. Then

$$
\begin{aligned}
a|p-q|+b|p-S q| & =\frac{1}{4}|p-q|+b\left|p-\frac{q}{5}\right| \\
& \geq \frac{1}{4}|p-q| \\
& \geq \frac{1}{5}|p-q| \\
& =|S p-S q|
\end{aligned}
$$

(iii) Suppose that $0 \leq q<1 / 515$ and $1 / 515 \leq p \leq 1$

$$
\begin{aligned}
a|p-q|+b|p-S q| & =a|p-q|+\frac{1}{4}|p-0| \\
& \geq \frac{1}{4}|p| \\
& \geq \frac{1}{5}|p| \\
& =|S p-S q| .
\end{aligned}
$$

(iv) Suppose that $0 \leq p<1 / 515$ and $1 / 515 \leq q \leq 1$

$$
\begin{aligned}
a|p-q|+b|p-S q| & =\frac{1}{4}|p-q|+\frac{1}{4}\left|p-\frac{q}{5}\right| \\
& \geq \frac{1}{4}\left|(p-q)-\left(p-\frac{q}{5}\right)\right| \\
& =\frac{1}{4}\left|\frac{4 q}{5}\right|=\frac{1}{5}|q|=|S p-S q| .
\end{aligned}
$$


Thus we conclude that $S$ is mean nonexpansive. Notice that, when $p=1 / 600$ and $q=1 / 515$, then $|S p-S q|>|p-q|$ and so $S$ is not nonexpansive. Choose $\alpha_{m}=0.70$ and $\beta_{m}=$ 0.65 , then the strong convergence and effectiveness of the Picard-Mann hybrid iteration can be seen in Table 1 and Figure 1.

\section{Conclusions}

We begun the finding of fixed points for mean nonexpansive operators in Banach spaces under the Picard-Mann hybrid iterative process. Some convergence results are established under different assumptions. It has been showen by an example that the notion of mean nonexpansive maps is properly more general than the notion of nonexpansive maps. Also, the Picard-Mann hybrid iterates of this example converge faster than the Picard, Ishikawa and Mann iterates. In particular, our results essentially improve and extended the results Khan [9] from the setting of nonexpansive operators to the larger frame work of mean nonexpansive operators.

\section{Data Availability}

No data were used to support this study.

\section{Conflicts of Interest}

We have no conflict of interest.

\section{Authors' Contributions}

Every author listed in this manuscript has contributed equally to each part.

\section{Acknowledgments}

The authors are grateful to the Spanish Government for Grant RTI2018-094336-B-I00 (MCIU/AEI/FEDER, UE) and to the Basque Government for Grant IT1207-19.

\section{References}

[1] S. Banach, "Sur les opérations dans les ensembles abstraits et leur application aux équations intégrales," Fundamenta Mathematicae, vol. 3, pp. 133-181, 1922.

[2] F. E. Browder, "Nonexpansive nonlinear operators in a Banach space," Proceedings of the National Academy of Sciences of the United States of America, vol. 54, no. 4, pp. 1041-1044, 1965.

[3] D. Gohde, "Zum Prinzip der Kontraktiven Abbildung," Mathematische Nachrichten, vol. 30, no. 3-4, pp. 251-258, 1965.

[4] S. S. Zhang, "About fixed point theory for mean nonexpansive mapping in Banach spaces," Journal of Sichuan University, vol. 2, pp. 67-78, 1975.

[5] C. X. Wu and L. J. Zhang, "Fixed points for mean nonexpansive mappings," Acta Mathematicae Applicatae Sinica, English Series, vol. 23, no. 3, pp. 489-494, 2007.

[6] Z. Zuo, "Fixed-Point Theorems for Mean Nonexpansive Mappings in Banach Spaces," Abstract and Applied Analysis, vol. 2014, Article ID 746291, 6 pages, 2014.
[7] J. Zhou and Y. Cui, "Fixed point theorems for mean nonexpansive mappings in CAT(0) spaces," Numerical Functional Analysis and Optimization, vol. 36, no. 9, pp. 1224-1238, 2015.

[8] S. Ishikawa, "Fixed points by a new iteration method," Proceedings of American Mathematical Society, vol. 44, pp. 147150, 1974.

[9] S. H. Khan, "A Picard-Mann hybrid iterative process," Fixed Point Theory and Applications, vol. 2013, no. 1, 2013.

[10] W. R. Mann, "Mean value methods in iteration," Proceedings of American Mathematical Society, vol. 4, no. 3, pp. 506-510, 1953.

[11] E. M. Picard, "Memorie sur la theorie des equations aux derivees partielles et la methode des approximation ssuccessives," Journal de Mathématiques pures et appliquées, vol. 6, pp. 145-210, 1890.

[12] S. Y. Cho, X. Qin, J.-C. Yao, and Y. Yao, "Viscosity approximation splitting methods for monotone and nonexpansive operators in Hilbert spaces," Journal of Nonlinear and Convex Analysis, vol. 19, pp. 251-264, 2018.

[13] Y. Yao, L. Leng, M. Postolache, and X. Zheng, "Mann-type iteration method for solving the split common fixed point problem," Journal of Nonlinear and Convex Analysis, vol. 18, pp. 875-882, 2017.

[14] Y. Yao, X. Qin, and J.-C. Yao, "Projection methods for firmly type nonexpansive operators," Journal of Nonlinear and Convex Analysis, vol. 19, pp. 407-415, 2018.

[15] J. A. Clarkson, "Uniformly convex spaces," Transactions of the American Mathematical Society, vol. 40, no. 3, pp. 396-414, 1936.

[16] Z. Opial, "Weak convergence of the sequence of successive approximations for nonexpansive mappings," Bulletin of the American Mathematical Society, vol. 73, no. 4, pp. 591-598, 1967.

[17] H. F. Senter and W. G. Dotson, "Approximating fixed points of nonexpansive mappings," Proceedings of American Mathematical Society, vol. 44, no. 2, pp. 375-380, 1974.

[18] J. Schu, "Weak and strong convergence to fixed points of asymptotically nonexpansive mappings," Bulletin of the Australian Mathematical Society, vol. 43, no. 1, pp. 153-159, 1991. 